\title{
Quantitative Electron-Excited X-ray Microanalysis at Low Beam Energy
}

\author{
Dale E. Newbury and Nicholas W. M. Ritchie \\ National Institute of Standards and Technology, Gaithersburg, MD 20899-8370
}

Quantitative electron-excited x-ray microanalysis performed at low beam energy $\left(\mathrm{E}_{0} \leq 5 \mathrm{keV}\right)$ offers significant advantages: (1) The linear dimensions of the electron interaction volume scale approximately as $\mathrm{E}_{0}{ }^{1.67}$ so that both lateral and in-depth spatial resolution are improved compared to the "conventional” beam energy range (10 keV to $30 \mathrm{keV})$. (2) Minimized x-ray absorption: As a direct consequence of the reduced beam penetration, x-ray absorption is significantly reduced and its impact on the analytical error budget is minimized. (3) For particle analysis, the greatly decreased electron range means that smaller particles can effectively be treated as bulk targets for quantitation. Instrumental advances have greatly improved low beam energy x-ray measurements: (1) Modern thermal field emission gun electron optics can deliver an analytically useful current into a beam diameter of $25 \mathrm{~nm}$ or less, minimizing the beam contribution to the limiting spatial resolution. (2) Large solid angle SDD-EDS systems, often consisting of arrays of detectors that can capture x-rays emitted over a broad range of azimuthal angle, enable collection of statistically robust spectra in reasonable measurement time that maximize the analytical information per unit electron dose. (3) The throughput of SDD-EDS enables collection of high count spectra, and the outstanding stability of SDD-EDS resolution and calibration with count rate provides the invariant peak shapes that enable successful multiple linear least squares peak fitting. Strongly interfering peaks can be accurately measured, even when the mutually interfering peaks have large ratios of relative intensity. Accurate SDD-EDS microanalysis performed with $\mathrm{E}_{0}$ from $5 \mathrm{keV}$ to $10 \mathrm{keV}$ of materials containing the low atomic number elements $\mathrm{B}$ to $\mathrm{F}$, which must be measured with photon energy peaks below $1 \mathrm{keV}$, has been demonstrated [1]. These advances suggest that it is now a propitious time to further explore the limits of quantitation at low beam energy. Low beam energy x-ray microanalysis is subject to several challenges: (1) While low beam energy SEM imaging can be performed with landing kinetic energies of a few hundred $\mathrm{eV}$ or even lower, the minimum useful beam energy for $\mathrm{x}$-ray microanalysis depends on the elements to be measured. For adequate excitation above the continuum background, it is desirable that the overvoltage, $U=E_{0} / E_{c}$, where $E_{c}$ is the critical ionization energy for the atomic shell of interest, is at least 2 for the highest $\mathrm{E}_{\mathrm{c}}$ to be measured. Values of $U$ in the range $1<U \leq 2$ are of course possible, but the peak-to-background decreases sharply below $U=2$. $E_{0}=5 \mathrm{keV}$ is the lowest beam energy at which there is a measureable characteristic peak for the entire Periodic Table, except $\mathrm{H}$ and He. (2) The range of x-ray production depends strongly on $\mathrm{E}_{\mathrm{c}}$ :

$$
\mathrm{R}(\mathrm{nm})=(27.6 \mathrm{~A}) /\left(\mathrm{Z}^{0.89} \rho\right)\left[\mathrm{E}_{0}^{1.67}-\mathrm{E}_{\mathrm{c}}{ }^{1.67}\right]
$$

It is useful to construct an $\mathrm{x}$-ray sampling depth axis parallel to the photon energy axis of the spectrum, as shown in Figure 1. In this case for 60Au-40Ag, $A_{u M}-N_{7}\left(A_{4}=2.21 \mathrm{keV}\right) \mathrm{x}-$ rays are created to a depth of $74 \mathrm{~nm}$, while $\mathrm{AgL}_{3}-\mathrm{M}_{5}\left(\mathrm{AgL}_{3}=3.35 \mathrm{keV}\right)$ are created to a depth of $48 \mathrm{~nm}$, meaning that there is a factor of 1.5 in linear dimension and 3.7 in volume over which the material being analyzed must be homogeneous to satisfy the basic requirement of the bulk quantification model. (3) The shallow sampling depth under low beam energy analysis conditions must be carefully considered when interpreting results. Surface oxide or other reaction layers and contamination that may be unnoticed at $\mathrm{E}_{0}=20 \mathrm{keV}$ can dominate the 
analysis results at low beam energy. This sampling depth effect is illustrated in Table 1, where the analysis of NIST SRM 481 (AuAg alloys) with various surface conditions is presented (kratio protocol with Au and Ag standards and NIST DTSA-II [2]). At a beam energy of $20 \mathrm{keV}$, the analysis of a polished but aged preparation of the four alloys shows anomalously low analytical totals but modest relative errors. Lowering the beam energy to $5 \mathrm{keV}$ reveals that a significant surface corrosion layer exists containing $\mathrm{S}$ and $\mathrm{Cl}$. After re-polishing to remove this layer, the analyzed values are found to correspond closely to the values on the SRM certificate.

[1] D. Newbury and N. Ritchie, Microsc. Microanal., 20 (Suppl 3) (2014) 702.

[2] N. Ritchie, DTSA-II available free at: www.cstl.nist.gov/div837/837.02/epq/dtsa2/index.html

Table 1

\begin{tabular}{|c|c|c|c|c|c|}
\hline Sample & Raw sum & S (norm.) & Cl (norm.) & Ag (norm.) & Au (norm.) \\
\hline 20Au80Ag_20keV & 0.9304 & Not detected & Not detected & 0.7747 & 0.2253 \\
\hline Relative error & & & & $-0.15 \%$ & $0.46 \%$ \\
\hline 40Au60Ag_20keV & 0.9610 & Not detected & Not detected & 0.5821 & 0.4179 \\
\hline Relative error & & & & $-2.9 \%$ & $4.4 \%$ \\
\hline 60Au40Ag_20keV & 0.9690 & Not detected & Not detected & 0.3787 & 0.6213 \\
\hline Relative error & & & & $-5.1 \%$ & $3.5 \%$ \\
\hline 80Au20Ag_20keV & 0.9722 & Not detected & Not detected & 0.1850 & 0.8150 \\
\hline Relative error & & & & $-7.3 \%$ & $1.8 \%$ \\
\hline 20Au80Ag_5keV & 0.9855 & 0.0934 & 0.1061 & 0.721 & 0.0796 \\
\hline Relative error & & & & $-7 \%$ & $-64 \%$ \\
\hline 40Au60Ag_5keV & 0.9959 & 0.0311 & 0.0965 & 0.5284 & 0.3440 \\
\hline Relative error & & & & $-12 \%$ & $-14 \%$ \\
\hline 60Au40Ag_5keV & 0.9951 & 0.0094 & 0.0450 & 0.3706 & 0.5754 \\
\hline Relative error & & & & $-7.2 \%$ & $-4.2 \%$ \\
\hline 80Au20Ag_5keV & 1.005 & 0.0051 & 0.0365 & 0.2244 & 0.734 \\
\hline Relative error & & & & $12 \%$ & $-8.3 \%$ \\
\hline 20Au80Ag_5keV_Repolish & 1.005 & Not detected & Not detected & 0.7602 & 0.2398 \\
\hline Relative error & & & & $-2 \%$ & $6.9 \%$ \\
\hline 40Au60Ag_5keV_Repolish & 0.9983 & Not detected & Not detected & 0.5955 & 0.4045 \\
\hline Relative error & & & & $-0.64 \%$ & $1.1 \%$ \\
\hline 60Au40Ag_5keV_Repolish & 0.9897 & Not detected & Not detected & 0.3916 & 0.6084 \\
\hline Relative error & & & & $-1.9 \%$ & $1.3 \%$ \\
\hline 80Au20Ag_5keV_Repolish & 0.9998 & Not detected & Not detected & 0.1946 & 0.8055 \\
\hline Relative error & & & & $-2.5 \%$ & $0.62 \%$ \\
\hline
\end{tabular}

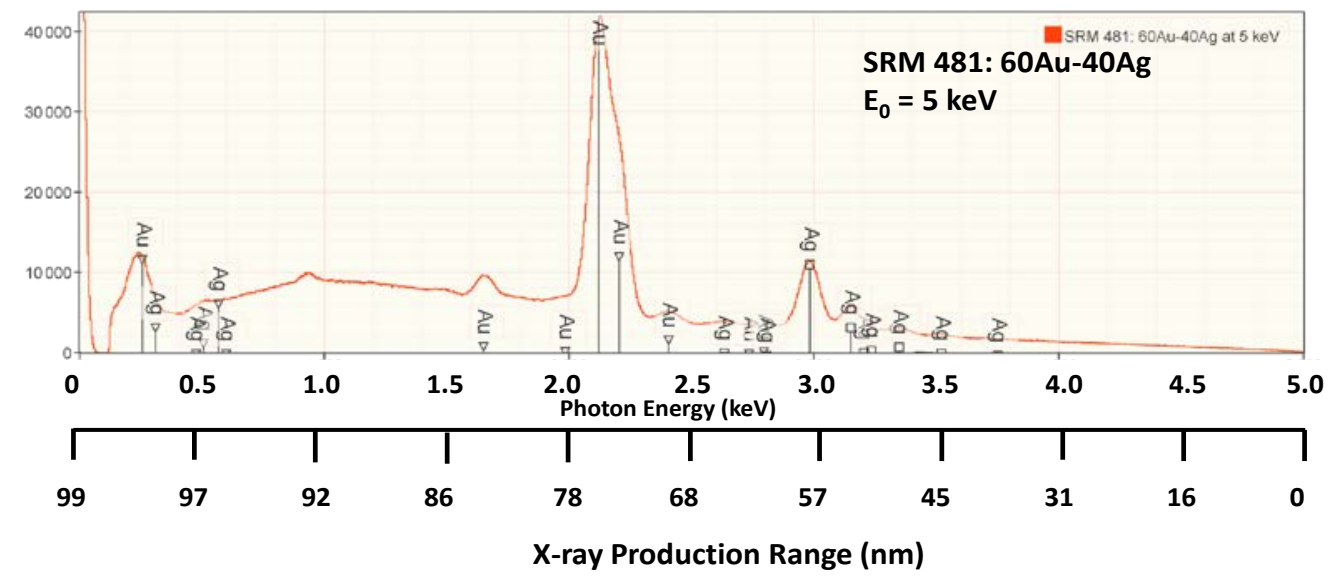

Figure 1. SDD-EDS spectrum of SRM481_60Au-40Ag at $E_{0}=5 \mathrm{keV}$ with depth of x-ray production. 